\title{
Le marché des produits vivriers et développement socio-économique dans l'Arrondissement de Sa'a (Région du Centre, Cameroun)
}

\author{
Kouna Binélé Marlise Sandrine \\ Institut National de Cartographie, Cameroun \\ Awono Mbassi Tatiana \\ Centre National de l'Education, Cameroun \\ Menyengue Eric François \\ Jakpou Njipnang Doris Nadine \\ Institut National de Cartographie, Cameroun \\ Mopi Touoyem Fabrice \\ Université de Yaoundé 1, Cameroun
}

Doi:10.19044/esj.2021.v17n16p72

Submitted: 10 February 2021

Accepted: 20 March 2021

Published: 31 May 2021

\author{
Copyright 2021 Author(s) \\ Under Creative Commons BY-NC-ND \\ 4.0 OPEN ACCESS
}

Cite As:

Marlise Sandrine K.B., Mbassi Tatiana A., François M.E., Doris Nadine J.N. \& Touoyem Fabrice M. (2021). Le marché des produits vivriers et développement socio-économique dans l'Arrondissement de Sa'a (Région du Centre, Cameroun). European Scientific Journal, ESJ, 17(16), 72. https://doi.org/10.19044/esj.2021.v17n16p72

\section{Resume}

L'économie rurale de bon nombre de pays en Afrique subsaharienne est essentiellement basée sur les activités agricoles. La crise économique des années 1980 qui a causé la baisse des prix des cultures de rente, a amené les agriculteurs à se lancer massivement dans les cultures vivrières. C'est ainsi que les campagnes sont devenues des lieux de ravitaillement pour les villes environnantes. L'objectif de cet article est d'évaluer l'apport du marché des produits vivriers au développement socio-économique de l'Arrondissement de Sa'a. Pour cela, l'étude s'est appuyée sur une recherche documentaire, des observations directes sur le terrain, des enquêtes par questionnaires auprès d'un échantillon de 425 acteurs intervenant dans la chaine de production, de transport, de commercialisation et de consommation des produits vivriers. Des entretiens semi-structurés ont aussi été réalisés avec les responsables des services déconcentrés du Ministère de l'Agriculture et du Développement Rural et les autorités de la Commune de Sa'a. Il ressort des analyses effectuées 
que le marché de Sa'a accueille un flux important de produits vivriers qui proviennent des différents bassins de production. Plusieurs acteurs interviennent à des différents niveaux dans le marché des produits vivriers à Sa'a. L'on a d'une part les commerçants parmi lesquels on retrouve les productrices-commerçantes, les immigrés saisonniers, les colportrices ou collectrices, les grossistes, les revendeuses et les détaillantes. D'autre part, l'on distingue les non commerçants qui regroupent les transporteurs, les consommateurs et les pouvoirs publics. Ces produits vivriers sont commercialisés à travers un circuit court, intermédiaire ou long. Leur commercialisation concerne beaucoup plus les femmes et génère des bénéfices qui vont de 3000 à 60000 FCFA pour les commerçantes, et 15000 à 35000FCFA en moyenne pour les transporteurs par moto taxi le jour du " grand marché ». Ces revenus enregistrés leur permettent d'augmenter leur capital et d'investir dans d'autres activités plus décentes et rentables. La Commune de Sa'a fait également des recettes importantes le jour du «grand marché». Les recettes collectées lui permettent de construire des infrastructures sociales. Il est donc important de le prendre en compte dans les politiques de développement parce qu'il représente un atout non négligeable pour le développement local.

Mot clés : Marché, produits vivriers, commerce, développement socioéconomique, Sa'a

\title{
Food market and socio-economic development in the Sa'a Subdivison (Centre Region, Cameroon)
}

\author{
Kouna Binélé Marlise Sandrine \\ Institut National de Cartographie, Cameroun \\ Awono Mbassi Tatiana \\ Centre National de l'Education, Cameroun \\ Menyengue Eric François \\ Jakpou Njipnang Doris Nadine \\ Institut National de Cartographie, Cameroun \\ Mopi Touoyem Fabrice \\ Université de Yaoundé 1, Cameroun
}

\begin{abstract}
Rural economies of many countries in sub-Saharan Africa are essentially based on agricultural activities. The economic crisis of the 1980s, which led to the drop in the price of cash crops, pushed farmers to massively move to food crops. As a result, rural areas became places where food crops
\end{abstract}


are cultivated in order to feed surrounding towns. The objective of this work was to assess the contribution of the food crops market to the socio-economic development of Sa'a Subdivision. So, the study was based on documentary research, field observations, and questionnaire surveys of a sample of 425 actors involved in the production, transport, trade and consumption of food crops. Semi-structured interviews were also carried out with local authorities of the Ministry of Agriculture and Rural Development and Sa'a council. The analyses carried out show that Sa'a market receives an important flow of food crops coming from various villages where they are produced. Several actors are involved at different levels in the food crops market in Sa'a. There are traders, who include producers-traders, seasonal immigrants, peddlers or collectors, wholesalers, resellers and retailers. There are also non-traders such as drivers, consumers and public authorities. Food crops are sold within short, intermediate and long circuits. The sale of food crops concerns particularly women and generates high incomes ranging from 3,000 to 60,000 CFA francs for traders, and 15,000 to 35,000 CFA francs on average for motorbike drivers on the day of the "big market". The collected incomes allow them to increase their capital or to invest in other activities which are more profitable. The Sa'a council also collects significant incomes on the day of the "big market". The incomes collected aid in the provision of social infrastructure for the population. It is therefore necessary to consider it in development policies as it represents a significant asset for local development.

Keywords: Market, food crops, trade, socio-economic development, Sa'a

\section{Introduction}

La crise économique intervenue à la fin des années 1980 a entrainé des bouleversements du système de production agricole en milieu rural en Afrique subsaharienne. La chute des cours mondiaux des cultures de rente, notamment ceux du cacao et du café, qui s'en est suivi, a poussé les agriculteurs à abandonner ces cultures d'exportation pour se tourner vers les cultures vivrières désormais plus rémunératrices, afin d'améliorer leurs revenus (Moupou et Mbanga, 2004 ; Ebela, 2017). Le développement du vivrier marchand se présente donc comme une réponse paysanne à cette situation de crise. Cette filière a connu un essor au fil du temps dans les campagnes camerounaises en général et celles de l'Arrondissement de Sa'a en particulier, grâce à la synergie de plusieurs acteurs aux logiques diverses.

De nos jours, l'expansion de ces cultures vivrières à Sa'a est également liée aux conditions naturelles très favorables, au développement des grandes villes, et à l'appui des pouvoirs publics qui a favorisé l'ouverture des fronts pionniers et de l'installation des migrants agricoles dans le Mbam et Kim voisin (Elong, 2004). Le marché des produits vivriers est devenu au fil des 
années une florissante dont vivent de nombreuses familles rurales et qui permet de ravitailler les grands centres urbains proches. Cette activité contribue également de manière significative au développement local à travers les recettes qu'elle génère à la Commune de Sa'a et dont cette dernière se sert pour améliorer le cadre de vie de ses populations. C'est ce qui justifie le choix porté sur ce travail intitulé : "Le marché des produits vivriers et développement socio-économique dans l'Arrondissement de Sa'a (Centre Cameroun)». Il est question, à partir d'une étude empirique et documentaire, d'analyser l'organisation et le fonctionnement du vivrier marcahand et d'évaluer ses retombées socio-économiques dans l'Arrondissement de Sa'a.

\section{Présentation de la zone d'étude}

Sa'a est un Arrondissement situé au Nord du département de la Lékié. Il est limité à l'Est par la Commune de Batchenga, au Nord et au Nord-Est par les Communes de Mbangassina et de Ntui, au Sud par la Commune d'Obala, à l'Ouest et au Sud-Ouest par les Communes d'Ebebda et de Monatélé. Ce territoire couvre une superficie de $546 \mathrm{~km}^{2}$ et compte 89 villages peuplés majoritairement par les Eton et Manguissa. Selon les chiffres du $3^{\mathrm{e}} \mathrm{RGPH}$ (2005), la densité de population de cette localité est de 91 habitants $/ \mathrm{km}^{2}$. Son milieu naturel est favorable aux activités agricoles .Son climat humide, caractérisé par des précipitations moyennes annuelles abondantes qui oscillent entre 1600 et $2000 \mathrm{~mm}$, ses sols ferralitiques profonds, son relief varié et son réseau hydrographique dense, sont favorables aux activités agricoles (figure $1)$. 
Figure 1: Localisation de l'Arrondissement de Sa'a

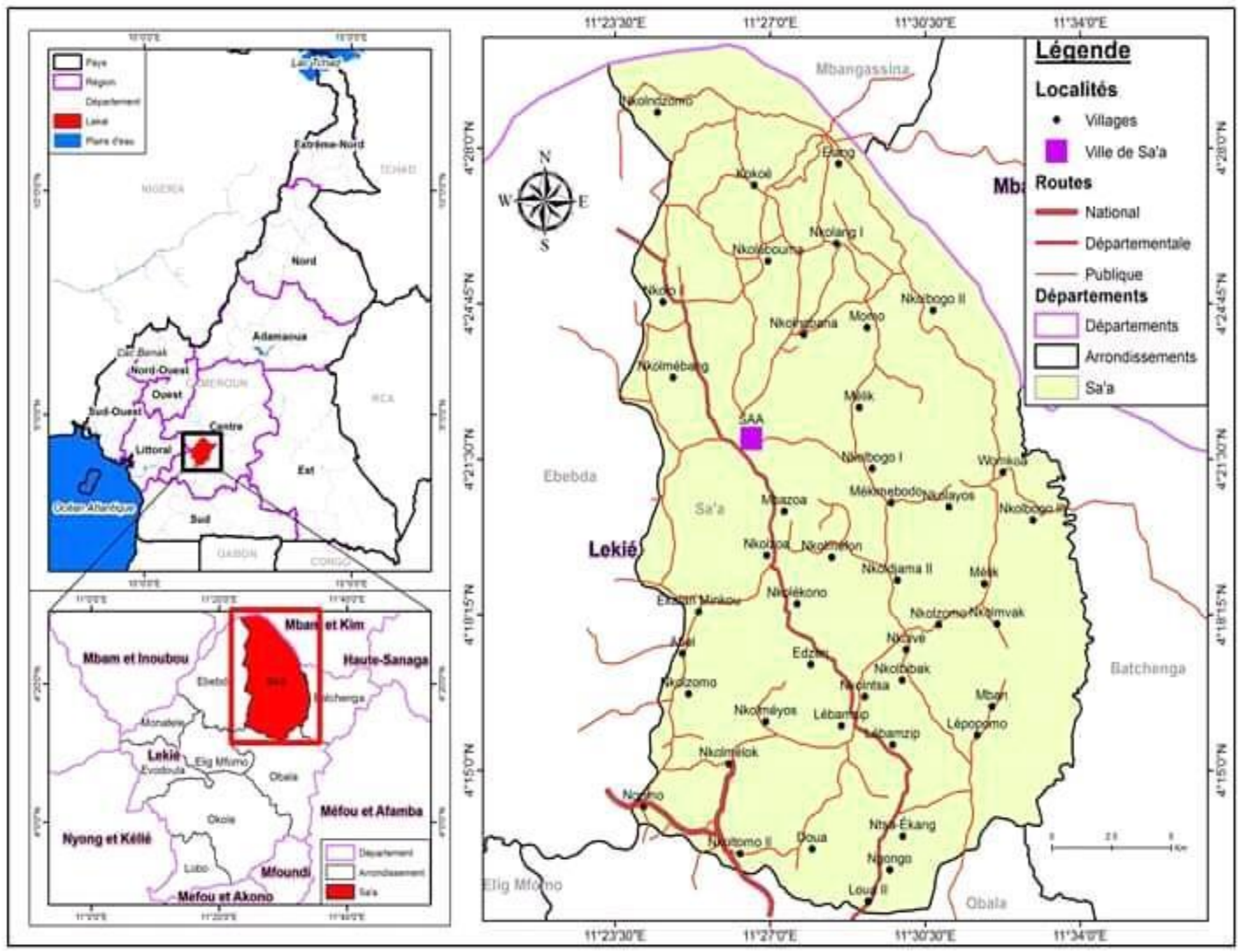

Source : Fond topographique feuille de Bafia au 1/50 000

\section{Méthodologie}

La méthodologie utilisée au cours de cette étude s'est appuyée, de prime abord, sur une recherche documentaire. Elle a permis de faire un tour de la littérature sur le marché des produits vivriers en Afrique subsaharienne en général et au Cameroun en particulier. Ensuite, des enquêtes par questionnaire ont été menées auprès de différentes catégories d'acteurs intervenant dans le marché des produits vivriers. Pour cela, un total de 425 questionnaires ont été administrés selon la technique d'échantillonnage stratifié. La taille de l'échantillon a été déterminée selon les critères définis par Nwana (1982), qui stipule que lorsque la population cible est de plusieurs centaines, $20 \%$ de celle-ci est représentative. Ainsi, 150 producteurs, 100 commerçants , 50 transporteurs tous types confondus et 75 ménages ont été enquêtés dans une population d'acteurs du vivrier marchand, répartie en 1500 individus et 375 ménages. Ceux-ci ont été approchés en prenant en compte leur degré d'implication dans la filière. Ces enquêtes avaient pour but de mieux comprendre les stratégies d'intervention de chacun, ainsi que les 
implications socio-économiques à Sa'a. Les observations directes sur le terrain quant-à elles, ont permis d'apprécier le niveau d'organisation et le fonctionnement de la filière. Les entretiens semi-structurés réalisés avec les personnes ressources dont : le délégué d'Arrondissement de l'Agriculture et du Développement Rural, les représentants des organisations de producteurs, des associations de commerçantes et de transporteurs, ont permis d'apporter un complément d'informations sur l'organisation et le fonctionnement de la filière, ainsi que les revenus générés par cette activité. Lesdites enquêtes ont été menées dans les marchés de Sa'a et de Banga, ainsi que dans 5 villages environnants séparés par une distance de 5 à $20 \mathrm{~km}$ environ (Nkol-Ebassimbi, Womkoa, Polo, Nkol-bogo II, Mbazoa). Le choix des sites d'enquête s'est opéré en se prenant en compte les lieux d'approvisionnement des différents marchés, l'ampleur de l'activité, l'accessibilité aux différents marchés, l'abondance de la clientèle et l'ancienneté de l'activité. Compte tenu de la rareté des statistiques officielles sur la production et la commercialisation des produits vivriers, parce que non encore imposables, les chiffres utilisés dans la présente étude sont ceux issus des enquêtes parcellaires sur les principaux marchés identifiés. Malgré le caractère diffus des circuits de distribution, ces statistiques permettent tout de même de montrer les implications socio-économiques du marché des produits vivriers dans l'Arrondissement de Sa'a.

\section{Résultats}

\section{Répartition des bassins de production}

Les produits vivriers sont cultivés dans tout l'Arrondissement de Sa'a, mais beaucoup plus dans certains bassins réputés comme : Nkol-Ebassimbi, Womkoa, Polo, Nkol-bogo II, et Mbazoa. Cependant, du fait de la pression foncière et la baisse de la fertilité des sols, les rendements sont devenus faibles. Par ailleurs, le département du Mbam-et-Kim fait aussi partie des pôles de ravitaillement du marché de $\mathrm{Sa}$ 'a en produits vivriers à cause de leur proximité et de la construction du «Pont de l'Enfance» dans les années 1970, qui relie ces deux zones. L'Arrondissement de Sa'a, en raison de sa position stratégique dans la Lekié, occupe une place de choix dans les échanges intérieurs et externes de produits vivriers marchands. Les rendements y sont significatifs et assez élevés. Certains producteurs rencontrés sur le marché central de Sa'a ont relevé que si l'on cultive un hectare d'ignames, l'on peut récolter une tonne, pour une valeur monétaire de près d'un (1) million de francs CFA. Que ce soit en période d'abondance ou de carence, le prix de $50 \mathrm{~kg}$ d'ignames oscille entre 25000 et 30000 FCFA. C'est sur la base des enquêtes menées auprès des commerça transporteurs que nous avons décelé le potentiel de ce grand bassin de production. Les commerçantes se ravitaillent soit dans les marchés périodiques comme ceux de Banga, Mvoundou etc., ou alors dans les 
villages-rue tels que: Endiguili, Mpi, Biakoa-ferme, Ngoro etc., où les producteurs viennent disposer leurs produits au bord de la route. Les conducteurs de moto taxi ou les chauffeurs de taxi de brousse, vont dans la majorité de ces villages transporter les marchandises pour les acheminer au marché centre de Sa'a. La répartition des différents bassins de production de la région en fonction de leur taille est présentée ci-dessous (figure 2).

Figure 2: Répartition des bassins de production à Sa'a

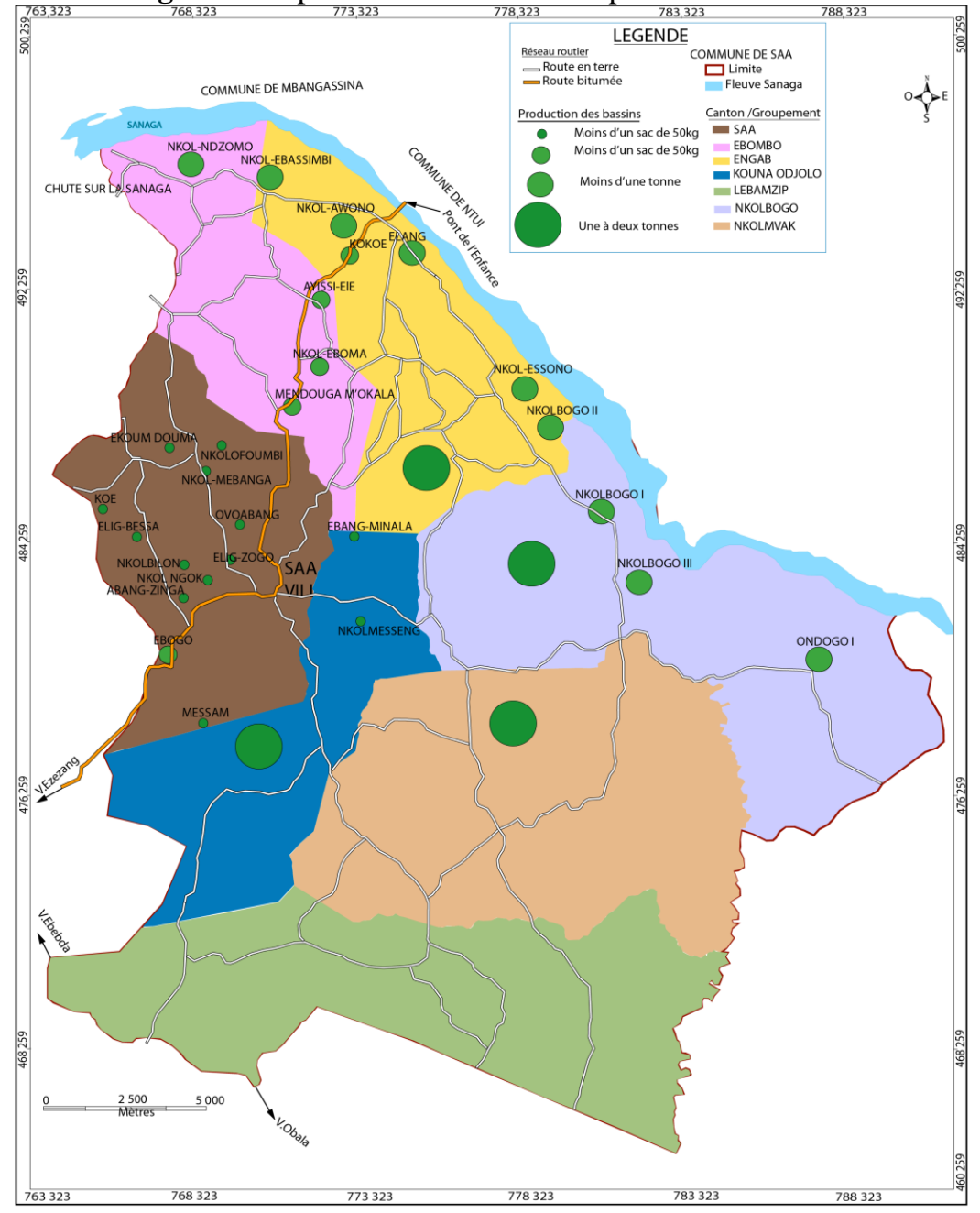

Source : Carte topographique de Bafia au 1/50 000

\section{Types de spéculations produites dans les bassins de production}

Les bassins de production qui ravitaillent le marché de Sa'a sont en proie à la diversification agricole. Plusieurs types de spéculations sont cultivées dans les bassins de production de l'Arrondissement de Sa'a. Parmi ces spéculations, l'on retrouve : les racines et tubercules, les céréales et oléagineux, la banane plantain et les légumes (tableau 1). 
Tableau 1: Types de spéculations cultivées dans l'Arrondissement de Sa'a

\begin{tabular}{|l|l|l|l|}
\hline \multicolumn{2}{|l|}{ Filières agricoles } & Appellation locale & $\begin{array}{l}\text { Période de } \\
\text { récolte }\end{array}$ \\
\hline \multirow{4}{*}{ Racines et tubercules } & Manioc & Nmoun & Plein temps \\
\cline { 2 - 4 } & Patate & Mebouda & Plein temps \\
\cline { 2 - 4 } & Macabo & Mebanga & Mars, avril. \\
\cline { 2 - 4 } & $\begin{array}{l}\text { Igname blanche, } \\
\text { igname sauvage }\end{array}$ & Djo'o, essol & $\begin{array}{l}\text { Novembre } \\
\text { décembre }\end{array}$ \\
\cline { 2 - 4 } & Taro & Mebanga mekomo & Décembre \\
\hline \multirow{2}{*}{ Céréales } & Maïs & Mbass & $\begin{array}{l}\text { Juillet, } \\
\text { août, } \\
\text { décembre }\end{array}$ \\
\hline \multirow{2}{*}{ Oléagineux } & Arachide & Owono & $\begin{array}{l}\text { Août, } \\
\text { décembre }\end{array}$ \\
\hline I & Pistache & Ngo 'on & Décembre \\
\hline Banane/plantain & Oignon du village & Medzan menam & Juin, juillet \\
\hline Légumes & $\begin{array}{l}\text { Plantain, banane } \\
\text { plantain }\end{array}$ & Ikon; odjo & Plein temps \\
\hline & Piment & ondoudoua & Plein temps \\
\cline { 2 - 4 } & Tomate, salades & / & Plein temps \\
\hline & Condiments & Bitjidga & Plein temps \\
\hline
\end{tabular}

Source : Enquête de terrain, janvier 2021

\section{Variation des prix de certaines denrées sur le marché local}

Les prix des différents produits vendus sur le marché local varient selon les lieux de vente et le nombre d'intermédiaires dans le circuit de commercialisation. Les prix bord champ proposés par les producteurs sont plus abordables par rapport à ceux fixés par les commerçants (tableau 2).

Tableau 2: Comparaison des prix de vente de certaines denrées

\begin{tabular}{|l|l|l|l|l|}
\hline $\begin{array}{l}\text { Produits } \\
\text { vivriers }\end{array}$ & $\begin{array}{l}\text { Unités de } \\
\text { mesure }\end{array}$ & $\begin{array}{l}\text { Prix de vente bord } \\
\text { champ (FCFA) }\end{array}$ & $\begin{array}{l}\text { Prix de vente } \\
\text { marché local } \begin{array}{r}\text { sur le } \\
\text { (FCFA) }\end{array}\end{array}$ \\
\hline Manioc & Sacs $(25 \mathrm{~kg})$ & 2000 à 2500 & 3500 à 5000 & \\
\hline Patate douce & Sacs $(50 \mathrm{~kg})$ & 2500 à 3000 & 4000 à 6000 \\
\hline Gombo & Sacs $(25 \mathrm{~kg})$ & 2500 à 3000 & 3500 à 5000 \\
\hline Igname & Cuvette $(50 \mathrm{~kg})$ & 25000 à 30000 & 35000 à 45000 \\
\hline Maïs & Sacs (50kg) & 3500 à 4000 & 5000 à 7000 \\
\hline Tomate & Cageot (25kg) & 3500 à 4000 & 4500 à 5000 \\
\hline Piment & Sceau (15 litres) & 1500 à 2000 & 2500 à 4000 \\
\hline
\end{tabular}

Source : Enquête de terrain, janvier 2021

Les prix des produits vivriers varient selon que l'on se trouve à Sa'a ou dans les marchés périodiques environnants. La diversité et la régularité de la clientèle, et la demande élevée sont les principaux facteurs qui régissent la fixation des prix bord champs à Sa'a.

Une fois le produit acheté par les grossistes, il transite entre les mains de plusieurs intermédiaires avant de parvenir au consommateur. Une fois 
les produits mis sur le marché, leurs prix varient d'un commerçant à l'autre, en fonction du moment de la journée (matin, midi, soir) et du prix d'achat (tableau 3).

Tableau 3: Prix de vente certaines denrées sur le marché local

\begin{tabular}{|l|l|l|l|l|}
\hline \multirow{2}{*}{$\begin{array}{l}\text { Produits } \\
\text { vivriers }\end{array}$} & Unité de mesure & \multicolumn{3}{|l|}{ Prix de vente (FCFA) } \\
\cline { 3 - 5 } & & Grossiste & Revendeur & Détaillant \\
\hline manioc & Sacs $(25 \mathrm{~kg})$ & 4000 & 4500 & 5000 \\
\hline Igname & Cuvette $(50 \mathrm{~kg})$ & 40000 & 45000 & 50000 \\
\hline Patate & & & \\
\hline Gombo & Sacs $(50 \mathrm{~kg})$ & 6000 & 6500 & $7000-8000$ \\
\hline Maïs & Sacs $(25 \mathrm{~kg}$ & 5000 & 5500 & $6000-7000$ \\
\hline Tomate & Sacs $(50 \mathrm{~kg})$ & 6000 & 7000 & 8000 \\
\hline Piment & Cageot $(25 \mathrm{~kg})$ & 5000 & 5500 & $6000-7000$ \\
\hline
\end{tabular}

Source : Enquête de terrain, janvier 2021

Les prix des denrées varient selon qu'ils se trouvent chez les grossistes, revendeurs et détaillants. Ceci s'explique par la simple raison que chacun à son niveau voulant obtenir un bénéfice, fixe les prix de vente en tenant compte du prix d'achat de la marchandise et des dépenses liées au transport. Les commerçantes grossistes se situent en amont de la chaîne de commercialisation. Elles incluent au prix de vente de la marchandise, le coût de transport de cette dernière, tout en tenant compte des marges bénéficiaires qui peuvent revenir aux revendeuses et détaillantes sur le marchélocal. Quant aux revendeuses, elles cherchent juste à obtenir un minimum de gain, en raison des multiples tracasseries dont elles font face à longueur de journée. Ce sont les détaillantes qui vendent généralement plus cher aux consommateurs, parce qu'elles se situent en aval de la chaîne de commercialisation. Elles vendent leurs marchandises en fonction du prix d'achat et des bénéfices qu'elles souhaiteraient avoir. Il faut d'ailleurs noter que tous les prix pratiqués par ces acteurs ne sont pas fixes. Ils varient en fonction des saisons et du rapport entre l'offre et la demande.

\section{Une demande élevée en produits vivriers}

L'augmentation de la population a entraîné une forte demande en produits vivriers. Cette population a pratiquement doublé en 15 ans en passant de 53219 habitants en 2005 selon les chiffres du 3ème RGPH, à pratiquement 105350 âmes en 2020. En effet, si la population camerounaise est restée en majorité rurale pendant les trois premières décennies après l'indépendance (environ $70 \%$ d'après les chiffres recensement général de la population de 1976). Cependant, la tendance s'est inversée au cours des dernières décennies durant lesquelles la population rurale s'est vidée pour se situer à environ $44 \%$ en 2017. Alors que le taux de croissance démographique annuelle est de 
l'ordre de $2,7 \%$, la population urbaine a augmenté de 5\% entre 1964 et 1976 et de 5,6\% entre 1976 et 1987 , pour se stabiliser autour de $5,4 \%$. La proportion de la population urbaine par rapport à la population totale est passée de 28,5\% en 1976 à 39\% en 1987 et $45 \%$ en 1995 (Hatcheu, 2003). Ce taux se situe aujourd'hui autour de $57 \%$. Face à cette croissance de la population, la demande en produits vivriers est devenue forte et le ratio des principaux produits vivriers pour 1000 habitants a été revu à la baisse (tableau 4).

Tableau 4: Evolution du ratio de la production des principaux produits vivriers pour 1000 habitants

\begin{tabular}{|l|l|l|l|}
\hline Spéculations & $\begin{array}{l}\text { Production moyenne } \\
\text { annuelle (tonnes) }\end{array}$ & $\begin{array}{l}\text { Ratio/1000 } \\
\text { habitants (2005) }\end{array}$ & $\begin{array}{l}\text { Ratio/1000 habitants } \\
\mathbf{( 2 0 2 0})\end{array}$ \\
\hline Arachide & 283 & 5,31 & 2,68 \\
\hline Maïs & 1208 & 22,69 & 11,46 \\
\hline Banane plantain & 32801 & 616,34 & 311,35 \\
\hline Banane douce & 1035 & 19,44 & 9,82 \\
\hline Huile de palme & 1106 & 20,78 & 10,49 \\
\hline Manioc & 42209 & 793,11 & 400,65 \\
\hline Macabo & 3676 & 69,07 & 34,89 \\
\hline Igname & 376 & 7,06 & 3,56 \\
\hline Patate & 43 & 0,80 & 0,40 \\
\hline Tomate & 488 & 9,16 & 4,63 \\
\hline Piment & 76 & 1,42 & 0,72 \\
\hline Gombo & 80 & 1,50 & 0,75 \\
\hline
\end{tabular}

Source : PCD de la commune de Sa'a et résultats du $3^{e} R G P H$

\section{Un vaste marché de consommation}

L'Arrondissement de Sa'a, de par sa position stratégique, reçoit un flux important de produits vivriers et accueille régulièrement des personnes d'origines diverses qui s'y rendent pour se ravitailler en produits vivriers. En effet, le marché central de Sa'a est un marché d'approvisionnement et de redistribution. C'est un lieu de transit des produits vivriers vers les grandes métropoles comme Douala, Yaoundé, et les villes frontalières de la Région du Sud (Ambam, Kye-ossi) ; y compris certains pays de la sous-région .Quatre facteurs le caractérisent comme marché de gros par excellence à savoir : le nombre élevé de grossistes (100 à 200), la gamme variée de produits vivriers vendus sur le marché (environ 12 spéculations), le tonnage annuel élevé des produits commercialisés ( 1 à 2 tonnes), et la permanence des flux de ces produits pendant toute l'année.

La clientèle qui fréquente ce marché est assez variée. On y distingue plusieurs catégories de clients à savoir : la population locale, les détaillantes, les revendeuses, les grossistes venues des grandes villes voisines, et les voyageurs. Les produits vivriers achetés sont destinés soit à la consommation directe, soit à la revente. Les voyageurs en transit qui s'y approvisionnent viennent d'horizons divers, et sont en majorité des citadins. 


\section{Les acteurs de la filière à Sa'a}

L'on distingue globalement deux catégories d'acteurs impliqués dans le marché des produits vivriers à Sa'a à savoir : les commerçants et les non commerçants.

\section{Les commerçants}

Ce sont les acteurs directement impliqués dans le commerce des produits vivriers. Parmi eux, l'on distingue : les productrices-commerçantes, les immigrés saisonniers, les productrices-colportrices, les colportrices ou collectrices, les grossistes, les revendeuses et les détaillantes.

\section{Les productrices-commerçantes}

Ce sont généralement des femmes, propriétaires d'exploitations agricoles, qui décident d'aller écouler elles-mêmes leurs produits au marché. En vendant leurs produits par leurs propres soins, elles ambitionnent gagner davantage qu'en les confiant aux colportrices ou en les écoulant sur place.

\section{Les immigrés saisonniers}

Ce sont des producteurs qui viennent s'installer de manière saisonnière à Sa'a afin de s'occuper de leurs plantations de cacao. Y étant, ils mettent leur temps à profit en se lançant parallèlement dans la production des cultures vivrières. Ils sont majoritairement constitués des personnes originaires de la Région du Nord-Ouest. Ceux-ci exploitent les bas-fonds marécageux pour la production des cultures maraîchères. Les principales cultures produites sont constituées de la tomate, du poivron, des légumes etc. Ces plantes sont privilégiées parce qu'elles ont une maturité précoce et sont commercialisables dans le court terme, c'est-à-dire au bout de 2 à 4 mois.

\section{Les productrices-colportrices}

Cette catégorie concerne les personnes propriétaires des champs qui, compte tenu des ressources limitées, ne font que de maigres récoltes. Pour cela, elles collectent les produits vivriers auprès d'autres consœurs productrices (sœurs, coépouses, belles-sœurs...) dans le village en faisant du porte à porte afin de compléter les marchandises qu'elles achemineront au marché.

\section{Les colportrices ou collectrices}

Elles englobent les femmes qui, bien que résidant au village, ne pratiquent pas l'agriculture, par manque de force physique ou de terres cultivables. Elles disposent généralement d'un capital financier important et préfèrent collecter les marchandises dans les domiciles ou les villages. Elles 
achètent la plupart de temps, les produits vivriers commercialisables à vil prix, les stockent, pour attendre le jour du marché afin de revendre aux détaillants, aux consommateurs et aux grossistes venus des grandes villes voisines. Pour cela, certains producteurs se méfient de ces femmes taxées «d'arracheuses de marchandises » qu'on appelle localement « alamra». Ces dernières expliquent qu'elles achètent les produits à un prix dérisoire parce que «le transport est cher, la collecte et le marchandage des produits dans les champs leur prennent assez de temps ». Néanmoins, certains producteurs apprécient ces femmes car, elles leur permettent d'éviter les pertes post- récoltes de certains produits périssables, compte tenu de la distance qui sépare le village du marché et de l'état des routes.

\section{Les grossistes}

Cette catégorie de commerçantes est constituée des productrices qui viennent elles-mêmes vendre leurs produits au marché ou alors des revendeuses en gros, généralement appelées «buyam-sellam », qui se rendent dans les marchés périodiques ou dans les villages pour acheter leurs marchandises. Celles-ci arrivent le plus souvent à la veille du jour du «grand marché» ou alors, le jour du «grand marché» même, qui se tient tous les jeudis. Elles livrent les marchandises aux revendeuses et aux détaillantes. Parfois elles-mêmes se transforment en détaillantes et vendent à un certain moment de la journée tà tout le monde, y compris aux consommateurs, étant donné que le marché de Sa'a accueille une gamme variée de clients venant des villes et villages environnants.

\section{Les revendeuses}

Elles sont à l'intermédiaire des grossistes et des détaillantes. Ce sont généralement des femmes qui prennent des marchandises auprès des grossistes dès leur arrivée au marché. Elles n'ont pas de comptoirs précis, raison pour laquelle la plupart d'entre elles stockent leurs marchandises dans des endroits précis ou alors, les consignent dans des véhicules. Considérées comme des demi-grossistes, leur prix d'achat est au-dessus de celui des grossistes. Ce sont elles les premières personnes à réquisitionner les produits venues des villages auprès des producteurs ou des grossistes. Ces revendeuses se confondent le plus souvent aux producteurs-commerçants. Elles se transforment parfois en détaillantes et vendent leurs produits en plein air sur le marché ou alors, les livrent aux détaillantes locales et acheteuses venues des centres urbains proches.

\section{Les détaillantes}

Elles vendent leurs produits sur place au courant de la semaine et se ravitaillent le plus souvent au marché de Sa'a. Certaines possèdent des 
comptoirs, mais le jour du marché, elles viennent exposer leur marchandise à l'air libre. La plupart d'entre elles n'ont pas assez de moyens pour acheter et conserver les produits en raison du caractère périssable de certains produits vivriers. Elles se ravitaillent auprès des grossistes ou des revendeuses. Ces détaillantes vendent les marchandises de troisième voire la quatrième main. Au fur et à mesure que le nombre d'intermédiaires dans la chaine augmente, le prix de vent subit une augmentation parce que chacun voudrait avoir un bénéfice à son niveau.

\section{Les non commerçants}

Ils regroupent les acteurs qui interviennent dans le marché du vivrier, mais ne sont pas directement impliqués dans les transactions commerciales des produits vivriers. Dans cette catégorie, l'on recense les transporteurs, les consommateurs et l'Etat.

\section{Les transporteurs}

Ce sont eux qui transportent les marchandises des lieux de production vers les marchés pour être écoulés. Malgré l'état de dégradation du réseau routier, ils se battent pour acheminer les vivres des bassins de production vers le marché central de Sa'a. Ces transporteurs utilisent différents moyens de transport pour mener cette activité. Ainsi, les moyens de transport les plus utilisés sont : la marche à pieds, les brouettes, les taxis de brousse, les pick-up etc. Mais depuis l'avènement du transport par moto taxi, le marché des produits vivriers a connu un souffle nouveau. Ces motos facilitent le transport des produits vivriers des zones reculées et difficiles d'accès vers les principaux marchés ou points de collecte. Parmi ces transporteurs, on retrouve aussi les chargeurs, pousseurs et porteurs. Ceux-ci facilitent le déplacement des marchandises d'un point à l'autre au niveau du marché.

\section{Les consommateurs}

Les consommateurs qui regroupent l'ensemble des ménages, interviennent en aval du circuit de commercialisation. Ils achètent les produits auprès des détaillants ou des producteurs pour la consommation familiale. Ceux-ci jouent un rôle très important dans le circuit économique parce que la demande et le prix de vente des produits vivriers sur le marché dépend de leur pouvoir d'achat. Lorsque la demande et le pouvoir d'achat sont faibles, cela affecte les revenus des producteurs et commerçants.

\section{L'Etat}

L'Etat intervient dans le marché des produits vivriers à Sa'a à travers ses démembrements. En effet, le Ministère de l'Agriculture et du Développement Rural (MINADER) octroie des subventions et offre des 
appuis divers en matériels et intrants agricoles aux producteurs pour booster la production. Il assure l'encadrement technique des agriculteurs à travers les postes agricoles. L'Etat régule également l'activité de transport public des personnes et des biens et perçoit les taxes sur le transport des produits vivriers. La Commune de Sa'a se positionne également comme un acteur important du marché des produits vivriers. Il intervient à travers la répartition des espaces marchands, le prélèvement des taxes, des droits de place, et des frais de nettoyage du marché. Elle participe aussi à la l'aménagement des routes qui desservent les bassins de production et finance les microprojets agricoles en octroyant des prêts à des coûts d'intérêts dérisoires aux porteurs de projets.

Le PNDP (Programme National de Développement Participatif) est une structure l'Etat qui accompagne les Commune ans le développement local. Il participe à travers la Commune de Sa'a au financement de la construction ou de la réhabilitation des infrastructures agricoles dans les différents bassins de production.

Chacun de ces acteurs qui précèdent joue un rôle important dans le fonctionnement de la filière à Sa'a (figure 3).

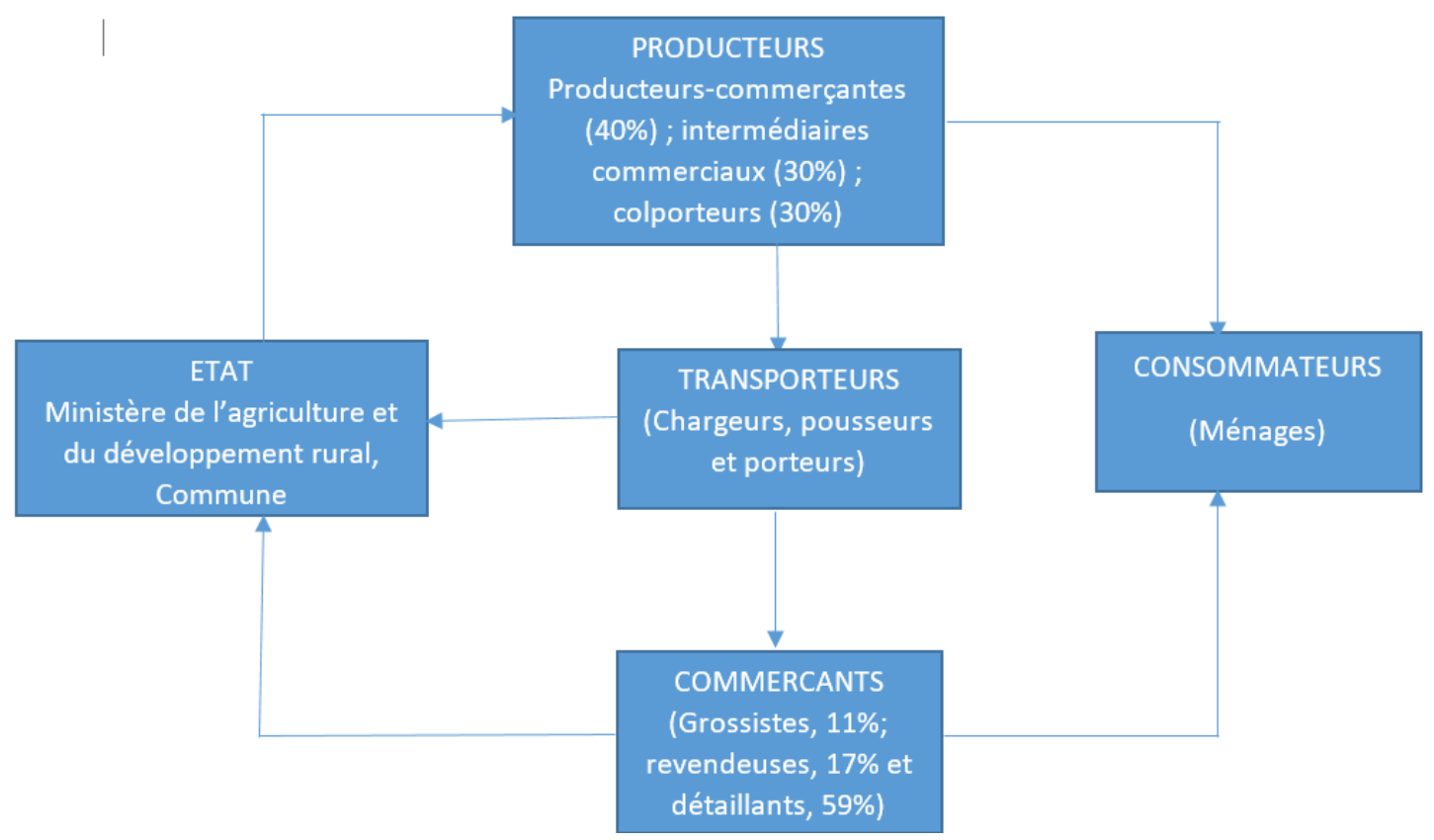

Source : Enquête de terrain, janvier 2021

Encadré : Les produits vivriers partent des producteurs pour atteindre les lieux de commercialisation à travers les transporteurs. Une fois acheminés dans les principaux points de commercialisation (marchés), consommateurs les . Néanmoins, certains consommateurs produits au niveau des producteurs. L'Etat quant-à lui finance et encadre les producteurs, prélève les taxes et impôts au niveau des transporteurs et commerçants. 


\section{Circuits de commercialisation}

D'après les observations faites sur le terrain, trois circuits types de commercialisation ont été identifiés à savoir : le circuit court, le circuit intermédiaire et le circuit long.

\section{Le circuit court}

Dans le cadre de ce circuit court, le produit commercialisé passe directement du producteur au consommateur. Les producteurs vendent directement leurs produits aux consommateurs qui les achètent, pour être consommés. A Sa'a, les producteurs qui récoltent ces produits de leurs champs, les transportent dans un véhicule, ou alors sur la tête et font la marche à pieds. Ils vendent généralement aux consommateurs à bas prix. Ceux qui adoptent ce type de circuit, représentent environ $15 \%$ et le font parce qu'ils cherchent à jouir entièrement du fruit de leur production.

\section{Le circuit intermédiaire}

C'est un circuit qui fait intervenir un seul intermédiaire entre les producteurs et les consommateurs. Ces intermédiaires sont très souvent constituées des revendeuses. Par ailleurs, à Sa'a, certaines revendeuses du marché central s'en vont parfois acheter leurs marchandises auprès des producteurs pour revendre aux consommateurs. Ainsi, ces marchandises qui proviennent directement des villages, sont acheminées vers les différents comptoirs à l'aide des porte-tout, des brouettes ou des véhicules.

Ces producteurs livrent habituellement en gros à ces revendeuses qui se chargent revendre en en tas pour permettre au consommateur qui le désire de trouver également son compte.

\section{Le circuit long}

Le circuit long quant-à lui, fait intervenir au moins deux agents économiques entre le producteur et le consommateur. A Sa'a, ce circuit consiste pour les producteurs à vendre leurs produits aux grossistes ou aux colportrices, qui vont à leur tour les revendre aux détaillantes chez qui les consommateurs viendront par la suite se ravitailler. Certains grossistes vont aussi chercher les marchandises dans certains marchés périodiques pour venir les livrer aux détaillantes au marché central de Sa'a.

\section{L'importance des flux des produits vivriers}

Les bassins de production qui ravitaillent le marché de Sa'a se caractérisent par l'importance des flux des denrées qu'ils évacuent. Le flux qui va du Mbam et Kim pour Sa'a représente $60 \%$ de la production, ensuite celui de Sa'a pour Yaoundé (30\%) et en fin les flux qui quittent de Sa'a vers Nkometou et Sa'a pour Obala (10\%). En effet, l'arrondissement de Sa'a 
recoit les flux des produits vivriers venant de l'ensemble de ses villages. Les produits qui viennent du Mbam et Kim y transitent avant d'être redistribués dans les grands centres urbains et les marchés frontaliers ou ceux de la sousrégion. Ces flux se présentent à Sa'a de la manière suivante (figure 4).

Figure 4 : Répartition des flux des produits vivriers

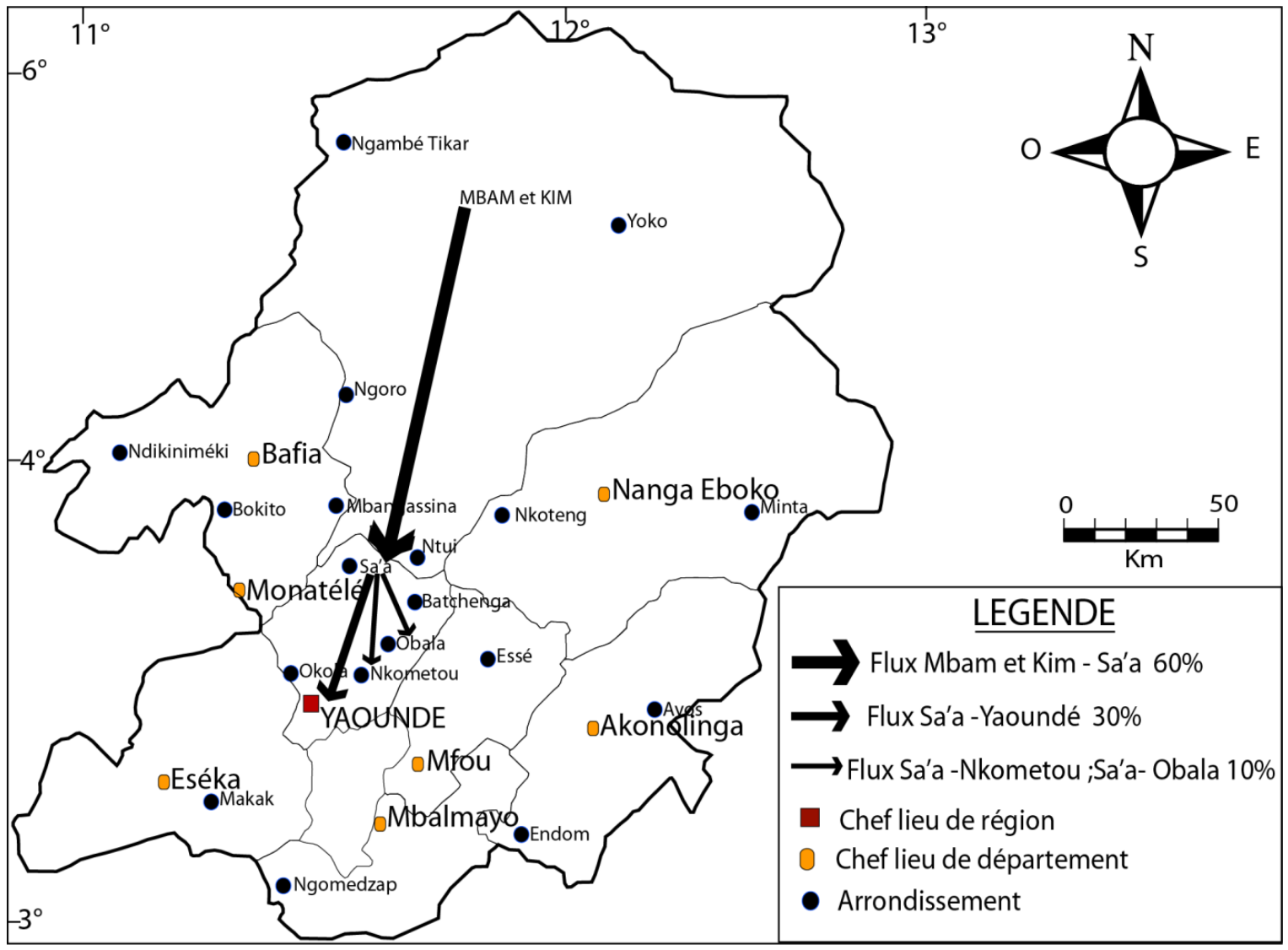

Source : Enquête de terrain, 2021

Implications socioéconomiques du marché des produits vivriers

Le marché des produits vivriers à Sa'a possède des enjeux économiques très importants. Ceux-ci sont liés aux bénéfices que génère cette activité à tous les acteurs impliqués à tous les niveaux de la chaine.

\section{Une amélioration sensible des revenus à travers les bénéfices réalisés}

Que ce soit au niveau de la production, de la commercialisation ou du transport des produits vivriers, chaque acteur s'en tire avec des bénéfices importants, dont le montant varie d'un acteur à un autre. Malgré le fait que beaucoup d'acteurs aient été réticents à déclarer leurs revenus, l'on peut quand même relever que les commerçantes réalisent des bénéfices proportionnels à leurs investissements et leurs statuts. 
Les détaillantes, après avoir revendu leur marchandise, réinvestissent l'argent gagné dans l'achat d'une nouvelle marchandise. Leurs bénéfices souvent faibles, se situent entre 3000 et 9000 FCFA par jour, à cause de leur fonds de commerce qui est généralement limité et des charges familiales qui, ne leur permettant pas toujours d'investir énormément dans cette activité Les grossistes et les revendeuses quant-à elles, réalisent des bénéfices hebdomadaires compris entre 9000 FCFA et 20000 FCFA, voire plus, parce qu'elles ont plus de capital que les détaillantes pour acheter les produits en grande quantité, raison pour laquelle elles gagnent parfois le double voire même le triple du prix d'achat de certaines marchandises. Les bénéfices réalisés le jour du «grand marché» par les différentes catégories de commerçantes se présentent de la manière ci-après (tableau 5).

Tableau 5 : Estimations des bénéfices réalisés par les commerçantes le jour du «grand marché» de Sa'a

\begin{tabular}{|l|l|}
\hline Catégories & Montants (FCFA) \\
\hline Grossistes & $20000-60000$ \\
\hline Détaillantes & $3000-9000$ \\
\hline Revendeuses & $9000-20000$ \\
\hline
\end{tabular}

Source : Enquête de terrain, janvier 2021

Quant aux transporteurs, ils font de gros bénéfices malgré l'état des routes et le prix élevé du carburant. Plusieurs moyens de transport sont utilisés pour transporter les produits. Les chauffeurs de taxis de brousse font le ramassage et parfois produisent des bénéfices inférieurs à ceux des conducteurs moto taxi. Leurs bénéfices se situent généralement entre 10 000 FCFA et 15000 FCFA par jour. Il faut aussi le relever que, les transporteurs qui gagnent le plus d'argent sont les conducteurs de moto taxi. Ceux-ci font généralement plusieurs navettes entre les bassins de production et le marché de Sa'a le jour du «grand marché», à cause de leur rapidité et de leur accès facile dans les coins les plus reculés où les routes sont parfois en mauvais état. (tableau 6).

Tableau 6 : Coûts estimatifs des bénéfices réalisés par les conducteurs de moto taxi le jour du «grand marché» de Sa'a

\begin{tabular}{|l|l|l|l|l|l|}
\hline Villages & Distance & $\begin{array}{l}\text { Moyen de } \\
\text { transport } \\
\text { utilisé }\end{array}$ & $\begin{array}{l}\text { Coût de } \\
\text { transport } \\
\text { avec } \\
\text { marchandises } \\
\text { (FCFA) }\end{array}$ & $\begin{array}{l}\text { Nombres de } \\
\text { tours /jour de } \\
\text { marché }\end{array}$ & $\begin{array}{l}\text { Recettes moyennes } \\
\text { journalières } \\
\text { (FCFA) }\end{array}$ \\
\hline Womkoa & $15 \mathrm{~km}$ & Moto taxi & 2500 & 15 tours & 37500 \\
\hline Nkol-bogo II & $15 \mathrm{~km}$ & Moto taxi & 2000 & 15 tours & 30000 \\
\hline $\begin{array}{l}\text { Nkol- } \\
\text { Ebassimbi }\end{array}$ & $15 \mathrm{~km}$ & Moto taxi & 2000 & 15 tours & 30000 \\
\hline Polo & $17 \mathrm{~km}$ & Moto taxi & 2500 & 10 tours & 25000 \\
\hline Mbazoa & $5 \mathrm{~km}$ & Moto taxi & 1500 & 15 tours & 22500 \\
\hline
\end{tabular}

Source : Enquête de terrain, janvier 2021 
La Commune pour sa part tire également son épingle du jeu dans ces transactions commerciales. Elle enregistre le jour du «grand marché», des recettes importantes provenant du paiement du ticket de marché par les commerçantes et les transporteurs qui desservent les différents villages. Elle se sert des sommes collectées pour entretenir le marché de Sa'a et améliorer le cadre de vie de ses habitants. Ces recettes collectées sont énormes et s'élèvent à environ 535000 FCFA par jour de marché (tableau 7).

Tableau 7 : Montant moyens des recettes collectées par la Commune de Sa'a le jour du «grand marché»

\begin{tabular}{|l|l|l|l|}
\hline Catégories d'acteurs & $\begin{array}{l}\text { Effectifs moyens } \\
\text { par jour de } \\
\text { marché }\end{array}$ & $\begin{array}{l}\text { Coûts unitaires du ticket de } \\
\text { marché(FCFA) }\end{array}$ & $\begin{array}{l}\text { Total } \\
\text { (FCFA) }\end{array}$ \\
\hline Grossistes & 150 & 1000 & 150000 \\
\hline Revendeuses & 200 & 1000 & 200000 \\
\hline Détaillantes & 170 & 500 & 85000 \\
\hline $\begin{array}{l}\text { Transporteurs } \\
\text { Total }\end{array}$ & 200 & 500 & 100000 \\
\hline
\end{tabular}

Source : Enquête de terrain, janvier 2021

\section{Les différents secteurs de réinvestissement des bénéfices réalisés}

La production, la commercialisation et le transport des produits vivriers ne sont qu'un tremplin pour bon nombre d'acteurs qui ne les considèrent que comme un moyen d'accumuler de l'argent pour réinvestir dans un autre secteur plus décent et rentable. La plupart de personnes interrogées ne souhaitent pas s'éterniser dans cette activité qu'ils devraient finir par abandonner tôt au tard. Pour cela, ceux-ci préparent la sortie de leurs activités en réinvestissant une partie de leurs gains dans d'autres secteurs plus rentables. Ceux qui ne pensent pas ainsi sont peu nombreux à savoir : les plus jeunes $(19,4 \%)$ et les personnes âgées (10\%). Certains chauffeurs de taxi rencontrés soit $9,7 \%$ des transporteurs, sont des étudiants qui exercent cette activité à titre conservatoire, en espérant une éventuelle réussite au concours d'entrée à la fonction publique ou dans les grandes écoles de formation.

Le domaine et la grandeur des investissements dépendent du type d'activité menée, du statut matrimonial, de l'âge et surtout de l'ancienneté dans le métier. Plus de la moitié des personnes inscrites dans une tontine $(60 \%)$ utilisent prioritairement l'argent épargné pour la construction ou la réhabilitation de leurs maisons, et subsidiairement pour l'achat d'un terrain. Parmi ceux ayant déjà investi ailleurs (75\%), les domaines d'activité dans lesquels ont porté leurs investissements sont : l'éducation des enfants, la santé familiale, l'immobilier, le transport et l'agriculture. Néanmoins, le secteur immobilier et l'achat d'un terrain sont les domaines dans lesquels ces acteurs 
investissent le plus, car $35 \%$ des investissements y sont consacrés. Ils sont suivis de l'éducation (22\%) et de l'épargne (28\%), (figure 5).

Figure 5 : Différents Secteurs de réinvestissement des bénéfices

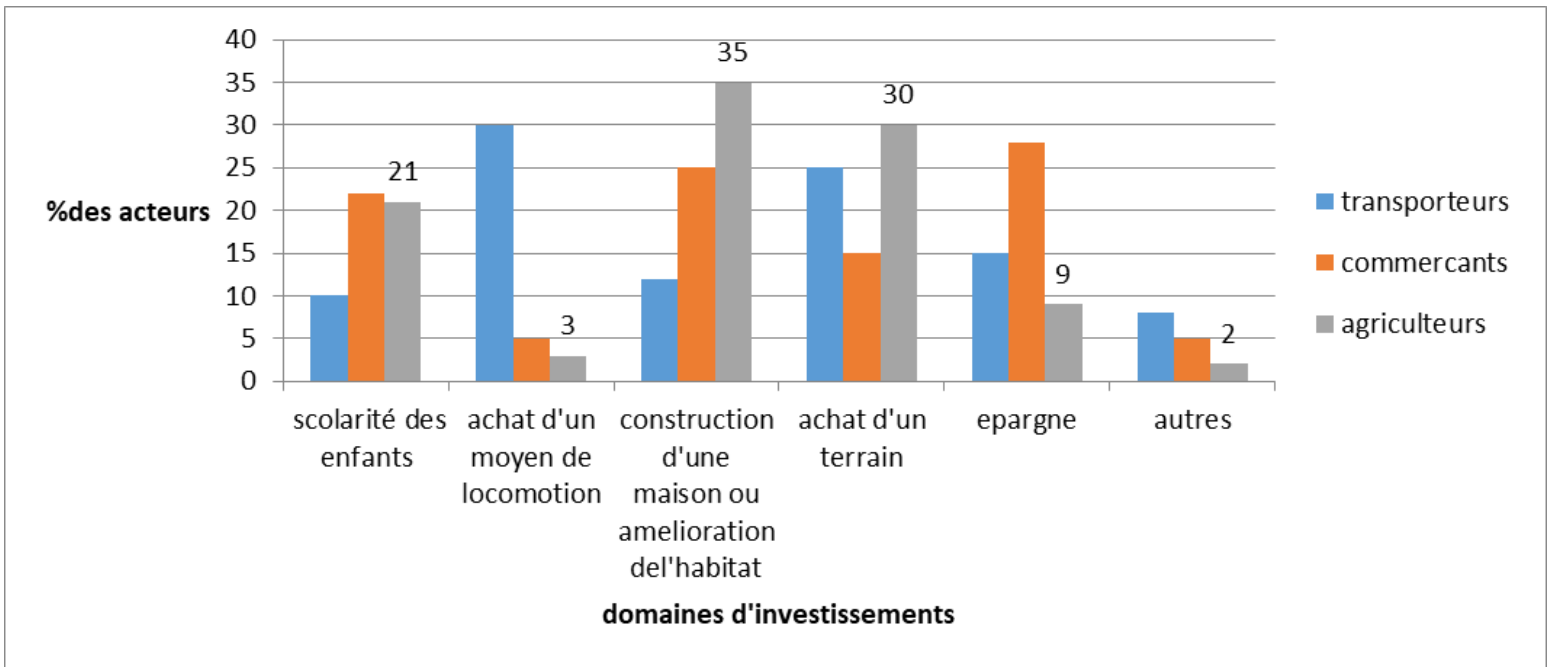

Source : Enquête de terrain, janvier 2021

La plupart des investissements réalisés dans l'immobilier concernent la construction des maisons d'habitation et la réhabilitation ou la réfection de maisons familiales.

Cependant, l'investissement dans l'achat des terres agricoles dans Mbam et Kim voisin est fréquent dans l'Arrondissement de Sa'a. Il concerne toutes les couches sociales confondues. L'économie de cet Arrondissement reposant essentiellement sur l'agriculture, l'achat des terres agricoles vise à créer des plantations pour accroitre les revenus.

En outre, $30 \%$ des transporteurs préfèrent plutôt réinvestir leur argent dans l'achat d'un nouveau matériel roulant. Ce type d'investissement concerne aussi une infime partie des producteurs. Ils se servent pour cela des revenus cotisés à partir des activités de vente et du transport des produits vivriers. La plupart des transporteurs qui acquièrent un nouveau matériel le font, tout simplement parce qu'ils veulent devenir autonomes, et ne plus travailler pour le compte d'une autre personne. Le moyen de transport généralement sollicité pour l'achat est la moto parce qu'il coûte moins cher par rapport à la voiture. Un producteur de gombo a confié à ce sujet avoir acheté deux motos en 1 an et les loue parfois à ses frères.

\section{Discussion}

Le développement de la filière vivrière n'est pas une particularité de l'Arrondissement de Sa'a. En effet, la crise économique de la fin des années 1980 a poussé les agriculteurs à mettre un accent sur les produits vivriers, 
consommés localement, au détriment des cultures de rente. C'est alors ainsi que, de nouveaux acteurs se sont intégrés dans le circuit de commercialisation de ces produits. C'est le même constat fait par Chaléard (2005) lorsqu'il montre qu'en Afrique de l'Ouest, et plus précisément dans les pays comme le Nigéria, le Ghana et la Côte d'Ivoire, le vivrier marchand tend à remplacer les cultures d'exportations. En milieu rural de nos jours, ces produits vivriers arrivent nettement en tête dans les revenus agricoles. Même si les revenus hebdomadaires restent modestes, le cumul annuel de ces revenus n'est pas négligeable. Dans l'Arrondissement de Sa'a, le marché des produits vivriers est un secteur d'activité qui nourrit de nombreuses familles. Les femmes particulièrement sont celles qui vivent de ce marché. Il faut tout de même noter que, l'essor du marché des produits vivriers est le fait de la synergie entre plusieurs acteurs qui interviennent à différents niveaux de la chaine de production, de transport et de commercialisation. Le même constat est observé par Kafo (2013) au sujet du commerce du vin de raphia qui s'organise autour des revendeuses et de nombreux intermédiaires commerciaux ayant intégré le circuit. De même, Goossens (1998) dans son étude a relevé la présence de deux ou trois intermédiaires dans le circuit de commercialisation. Les résultats de son étude corroborent également avec ceux observés à Sa'a, lorsqu'ils montrent que, la plupart des grandes villes africaines ne possèdent pas de véritables marchés de gros. Dans ces villes, les achats en gros des produits vivriers sont assurés par des colporteurs dans les champs, en bordure de route, dans les fermes, et éventuellement dans les différents marchés des villages abritant les bassins de production, mais rarement dans d'autres marchés ruraux. cet auteur continue en soutenant sur la base de plusieurs études de cas que, le commerce de gros se concentre en majorité sur les vivres hautement standardisés, pour lesquels l'information sur les prix et la qualité est bonne, le marchandage non nécessaire, et les transactions efficaces et faciles à contrôler. Cette réalité est similaire à celle observée au cours de cette étude, sauf qu'à Sa'a, les prix de ces produits vivriers connaissent une forte variation saisonnière des prix, et même d'un commerçant à un autre au sein du même marché et nécessite un marchandage pour les acheter à un prix relativement abordable.

Par ailleurs, les marchés de Sa'a se caractérisent par leurs dimensions et la diversité des fonctions qu'ils assurent. Ils sont pour cela comparables au marché d'Abobo-Centre présenté par Dakouri ( 2015), non seulement comme des lieux de rencontre des populations venues d'horizons divers, mais aussi comme des espaces d'échange des produits de gros, de demi-gros ou de détails.

Le marché des produits vivriers à Sa'a est aussi fortement influencé par les coûts du transport, la variation des prix qui sont régis par la loi de l'offre et de la demande, et l'état des voies de communication. Ceci coïncide 
avec les résultats obtenus par Tognide (2019) qui montrent que, les facteurs de performance du marché des produits vivriers sont liés à divers facteurs notamment: le manque d'information sur les prix des produits dans les marchés $(97 \%)$, l'usage de diverses unités de mesures (78\%), la mauvaise qualité du réseau routier (46\%), le coût élevé du transport (97\%), le rapport entre l'offre et la demande (72\%), et la fixation des prix (70\%).

Par ailleurs, ce marché des produits vivriers à Sa'a permet de subvenir aux besoins de nombreux ménages, grâce aux revenus qu'il rapporte. Les travaux réalisés par Ebela (2017) dans le département de la Mvila au Sud du Cameroun rejoignent ce constat lorsqu'ils montrent que, le vivrier marchand occupe une place non négligeable dans les revenus des ménages agricoles $(40 \%)$. Il permet à de nombreuses familles ceux-ci de gérer certaines charges familiales à côté des revenus du cacao.

\section{Conclusion}

L'analyse de l'apport du marché des produits vivriers au développement socio-économique dans l'Arrondissement de Sa'a a permis de relever que les conditions naturelles favorables ont favorisé la diversification agricole dans les différents bassins de production de cette région. La forte demande en produits vivriers et la présence d'une clientèle variée ont suscité le développement d'un marché plus ou moins organisé autour des produits vivriers à Sa'a. Plusieurs types acteurs travaillant en synergie, s'activent au quotidien dans l'organisation et le fonctionnement de ce marché dont vivent de nombreuses familles. Ces acteurs, comprennent d'une part les acteurs marchands constitués essentiellement des producteurs et des commerçants et d'autre part les acteurs non marchands qui regroupent les autres intervenants de la filière qui ne sont pas directement impliqués dans le commerce des vivres. Le marché des produits vivriers à Sa'a génère des bénéfices importants au regard du flux important des produits vivriers qui transitent sur le marché de la localité.

Les bénéfices que génère cette activité sont à l'origine des investissements parfois importants. A cet effet, les commerçantes enregistrent des bénéfices qui varient entre 3000 FCFA et 60000FCFA le jour du «grand marché», et entre 22000FCFA et 37000FCFA pour les conducteurs de moto taxi. Ces bénéfices sont généralement réinvestis dans d'autres activités plus décentes et rentables, ou alors, utilisés pour subvenir aux besoins familiaux. S'agissant de la Commune de Sa'a, elle encaisse également des recettes importantes le jour du «grand marché», dont les montants s'élèvent en moyenne à 500000 FCFA. Ces recettes ainsi collectées lui permettent d'aménager le cadre de vie des populations et de contribuer au développement local. 
L'appui au développement de la filière vivrière peut donc être envisagé pour lutter contre la pauvreté en milieu rural.

\section{References:}

1. Aboudrare, A., (2014). Importance socio-économique du safran pour les ménages des zones de montagne de la région de TaliouineTaznakht au Maroc, in : Revue marocaine des sciences agronomiques et vétérinaires, vol.2, $\mathrm{n}^{\circ} 1$, pp5-14.

2. Aka, K. A., (2013). Le circuit de distribution de l' "Attiéké » à Abidjan : typologie de la chaine de transport et dynamisme des acteurs, Centre de Recherches Architecturales et Urbaines (CRAU) in: Revue de géographie de l'université de Ouagadougou, $n^{\circ} 2$, pp $101-$ 121.

3. Anonyme, (2017). Rapport sur les produits de base et le développement des marchés des produits de base, 111p.

4. Bado, A., (2006). Opportunités sociales et économiques et développement du vivrier marchand à Bouaké (Côte d'Ivoire), in : Cahiers d'Agriculture, vol.15 n³, pp 33-37.

5. Courade, G., (2000). Le désarroi camerounais, Paris, Karthala ,286p.

6. Dakouri Guissa, D.F., et Koulaï, A., (2015). Commercialisation des produits vivriers et la dégradation de l'environnement dans les marchés d'Abobo-Centre (Abidjan- Côte d'Ivoire), in: Revue de géographie tropicale et d'environnement, $\mathrm{n}^{\circ} 1$, pp 66-76.

7. Diakalia, (2015). Evaluation de l'inflation des prix des produits vivriers dans la Commune de Yopougon (Abidjan-Côte d'ivoire) in : European Scientific Journal, vol.11, n², pp218-23.

8. Douka, A. M., (2011). Coopératives de vivriers et organisation spatiale à Abobo, in: Revue de géographie tropicale et d'environnement, $\mathrm{n}^{\circ} 1 \mathrm{pp} 31-43$.

9. Ebela, P. A., (2017). Le vivrier marchand dans la lutte contre la pauvreté des ménages en milieu rural : le cas du département de la Mvila dans le Sud du Cameroun », thèse de doctorat en géographie, Université de Bordeaux, $381 \mathrm{p}$.

10. Elong, J.G. (2004), Eton et Manguissa, de la Lékié au Mbam-et-Kim : Jeux et enjeux fonciers, in : Cahiers d'Outre-Mer [en ligne], pp 226227 avril-septembre, mis en ligne le 13 février 2008. Url : http://com.revues.org/index $572 . \mathrm{htm}$

11. FAO (2010). L'état de l'insécurité alimentaire dans le monde: Combattre l'insécurité alimentaire lors des crises prolongées, Rome, 150p.

12. Goossens, F., (1998). Commercialisation des vivres locaux: Le secteur informel dans une perspective dynamique, programme 
d'approvisionnement et de distribution alimentaires des villes Collection, aliments dans les villes, $92 \mathrm{p}$.

13. Hatcheu Tchawe, (2003). L'approvisionnement et la distribution alimentaire à Douala (Cameroun): Logiques sociales et pratiques spatiales des acteurs, Thèse de doctorat, Université de Paris I Panthéon Sorbonne ,454p

14. Kaffo, C., (2013). Ouest-Cameroun, valorisation des produits locaux: L'exemple du vin de raphia, in: revue économie rurale, $\mathrm{n}^{\circ} 336, \mathrm{pp}$ 61-79.

15. Kengne, F., (2002). Les producteurs ruraux dans la crise au Cameroun la province du centre, Paris, Harmattan ,258p.

16. Kouna Binele, M. S., (2014). Le vivrier marchand dans l'arrondissement de Sa'a : Stratégies des acteurs, organisation et fonctionnement de la filière, Mémoire de Master II en géographie, UYI, 150p.

17. Magrin, G. (2000). Vivrier marchand et intégration régionale : L'essor de la culture de l'arachide au sud du Tchad, CIRAD-PRASAC, Université de Paris I, 65p.

18. Mamadou Gbongué, (2017). Actualisation de la liste des produits vivriers susceptibles d'être commercialisés avec succès au travers de la bourse régionale des produits vivriers (BRPV), rapport final, $92 \mathrm{p}$.

19. Mohamed Moussaoui, (1994). Analyse socio-économique des rôles de l'agriculture et conséquences en matière de politiques : Etude de cas du Maroc, projet FAO - ROA /Maroc, CIRAD, province des iles, Nouvelle Calédonie.

20. Moupou, M. et Mbanga, L.A. (2008). Désengagement de l'Etat et réponses paysannes au Cameroun, in : Cahiers d'Outre-Mer, vol. 1-2, $n^{\circ}$ 241-242, pp163-183.

21. Musabanganji, E., (2017). Contraintes et stratégies d'amélioration de la filière maïs au Rwanda, Communauté Française de Belgique, Université de Liège - Gembloux agro-bio Tech $251 \mathrm{p}$.

22. Ombolo, J.P., (1998). Dictionnaire des villages de la Lékié.

23. Tognide, A. H., (2019). Circuits et performance de commercialisation des produits vivriers de la dépression de la lama ( Communes de Toffo et Zogbodomey) au Sud-Bénin, in : Afrique science, vol.15, $\mathrm{n}^{\circ} 3$ pp $142-159$. 